Robotica: (2021) volume 39, pp. 55-71. (C) The Author(s) 2020. Published by Cambridge University Press. This is an Open Access article, distributed under the terms of the Creative Commons Attribution licence (http://creativecommons.org/ licenses/by/4.0/), which permits unrestricted re-use, distribution, and reproduction in any medium, provided the original work is properly cited.

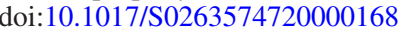

\section{A Review of Dynamic Balancing for Robotic Mechanisms}

\section{Bin Wei*๑ and Dan Zhang}

\author{
York University, 4700 Keele Street, Toronto, ON M3J 1P3, Canada \\ E-mail: dzhang99@yorku.ca
}

(Accepted 13 February 2020. First published online: March 11, 2020)

\begin{abstract}
SUMMARY
The authors summarize the main dynamic balancing methods of robotic mechanisms in this paper. The majority of dynamic balancing methods have been presented, and there may be other dynamic balancing methods that are not included in this paper. Each of the balancing methods is reviewed and discussed. The advantages and disadvantages of each method are presented and compared. The goal of this paper is to provide an overview of recent research in balancing. The authors hope that this study can provide an informative reference for future research in the direction of dynamic balancing of robotic mechanisms.
\end{abstract}

KEYWORDS: Robotics; Review; Automation; Design; Manufacturing.

\section{Introduction}

When mechanisms conduct operations, in some cases their mass center and angular momentum change, and vibration is thus generated. Dynamic balancing involves force balancing and moment balancing. Force balancing can be realized through having the center of mass (CoM) of the mechanism settled at a spot. Moment balancing can be realized through letting the angular momentum be constant. The shaking force balancing and shaking moment balancing of mechanisms can be traced back to 1960 s in scholars Berkof and Lowen's works. ${ }^{1-3}$ After that, some significant papers have been published in this area. For example, Ricard and Gosselin published a paper on the reactionless conditions studied for robot mechanisms in $2000 .{ }^{4}$ From that point, the study of dynamic balancing began to emerge in the academic research arena of robotics.

In many engineering applications, having a dynamic balancing condition is crucial, for example, the robotic mechanisms used in space. ${ }^{5}$ If one does not have a dynamic balancing condition, it will remarkably deteriorate the mechanism capabilities. ${ }^{6-8}$ For the purpose of accomplishing dynamic balancing, one needs to fulfill both force balancing and moment balancing. Usually counter-masses are used for realizing force balancing, and counter-rotations are usually employed to realize moment balancing. The question of achieving force balancing and moment balancing can be summarized as: typically employing additional components (rather than removing mass) to balance the original unbalanced conditions. However, when one uses additional balancing components, the system becomes heavier, which means more energy is needed to drive the system, which is not cost effective. There are, of course, many other methods that are able to achieve a dynamic balancing condition, which are reviewed and discussed here, and this is the main theme of this study.

Before the authors proceed further, it is necessary to reiterate the dynamic balancing conditions in a single plane. In order to achieve the dynamic balancing, one usually needs to have the following conditions (the linear momentum and angular momentum are constant):

$$
\begin{array}{r}
m \cdot v=m \cdot \dot{x}_{\text {com }}=P \rightarrow \text { constant } \\
I \cdot \omega=L \rightarrow \text { constant }
\end{array}
$$

* Corresponding author. E-mail: binwei28@yorku.ca 


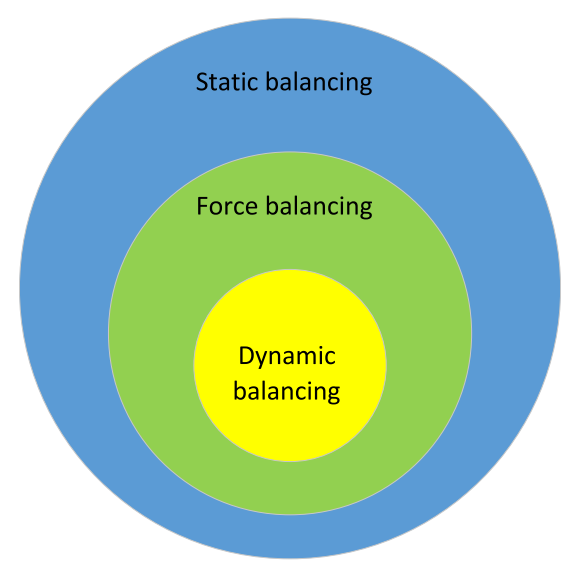

Fig. 1. Relationship between static balancing, force balancing, and dynamic balancing.

where $P$ is the linear momentum, $x_{\text {com }}$ is the position of $\mathrm{CoM}$, and $L$ is the angular momentum. From a different perspective, it is known that a mechanism is dynamically balanced when the sum of all forces and moments acting on the base are zero, ${ }^{9}$ that is,

$$
\begin{gathered}
F=m a=m \frac{d v}{d t} \\
M=\frac{d L}{d t}
\end{gathered}
$$

where $L$ is the angular momentum. Noted that the equations above for momentum and angular momentum balance are for the case that it is in 1D. Whereas for angular momentum balance in $3 \mathrm{D}$ case, if the same equation is used, it must be in the spatial frame, where $I$ is not constant. If it is expressed in a body-fixed frame, then $I$ is constant, and then the equation will relate to the Euler's equation of motion by including one extra term. If the right-hand side of the above two equations remains zero during motion, then the force and moment (acting to the base) will be zero, which means dynamically balanced/reactionless. Thus, we have the following two conditions for having a reactionless condition:

$$
\begin{aligned}
& \text { Center of mass } \rightarrow \text { constant }(\text { fixed) } \\
& \text { Angular momentum } \rightarrow \text { constant }
\end{aligned}
$$

Another interesting fact regarding the relationship between the static balancing, force balancing, and dynamic balancing can be illustrated in Fig. 1. Dynamic balancing is a subset of force balancing, and force balancing is a subset of static balancing. It is important to distinguish between static balancing and forcing balancing. Static balancing is the case where motors do not have to counter to the weight of the system, and the system can still remain stable in any configurations,${ }^{10}$ which can be achieved by using counter-masses, springs, or friction. ${ }^{11}$ Static balancing conditions can be achieved by making the potential energy of the system equal zero. Whereas force balancing is the case where the net force acting on the base is zero. If a system is force balanced, it is also static balanced; but if a system is static balanced, it does not necessarily mean that it is force balanced.

In ref. [12], dynamic balancing is mainly categorized into two families: "balancing prior to kinematic synthesis" and "balancing after the design." In this paper, the dynamic balancing methods based on these two classes are studied. This study is essentially an extension of the authors' previous presented paper in the 2016 ASME International Design Engineering Technical Conferences and Computers and Information in Engineering Conference. ${ }^{13}$

For dynamic balancing after the design, adding separate counter-masses and counter-rotations, adding special four-bar linkages, adding springs and counter-masses together, adding counterrotary counter-mass (CRCM), adding active-driven CRCM, adding active dynamic balancing block (ADBB), adding auxiliary links, and balancing through trajectory planning and control are presented in detail. Fisher's method falls into the category of balancing prior to kinematic synthesis. 

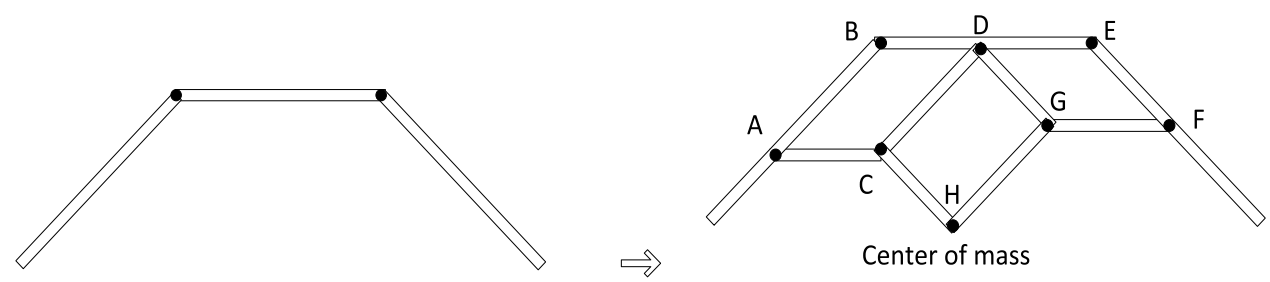

Fig. 2. Principal vector structure.

The paper is organized as follows: Section 2 presents the methods under the balancing prior to kinematic synthesis; balancing after the design methods are presented in Section 3; Section 4 compares each balancing method and lists od advantages and disadvantages of each method; the impact of dynamic balancing and future directions are presented in Section 5; and Section 6 gives the conclusion.

\section{Balancing Prior to Kinematic Synthesis}

\subsection{Fisher's method}

In 1906, the physician Otto Fisher proposed the approach of adding auxiliary parallelograms to the system to trace the CoM, as shown in Fig. 2. All the linkages are connected by revolute joints in this paper, unless otherwise stated. The dots in Fig. 2 below represent revolute joints.

The main idea of the Fisher's method is that by adding auxiliary parallelograms to the system, one can make the center of the mass fixed at a stationary point H, as shown in Fig. 2. Details can be referred to ref. [14]. A practical application of Fisher's method can be found in force balancing of Delta robot. ${ }^{12}$ In working leading to this application, scholar V. Wijk investigated and employed this approach, developing a methodology for analysis and synthesis for inherently balanced mechanisms. ${ }^{15}$ The pantograph structure is used to achieve the dynamic balancing. Regarding the pantograph structure, the linear momentum is first resolved, after which the force balance conditions are determined. Finally, the principal dimensions can be derived. Due to the fact that the 2-degree-of-freedom (DOF) pantograph structure does not contain a center linkage, one can determine the principal dimensions without resorting to the equivalent linear momentum systems (ELMS). Since the 3-DOF and 4-DOF principal vector structures have center chains, the ELMS are employed to determine the principal dimensions. Wijk ${ }^{15}$ used Fisher's methodology in his work to compute the principal dimensions and by using the pantographs to settle the CoM of the mechanism at a stationary point. The results indicate that the principal vector linkage structure is force balanced. Regarding the moment balancing issue, the movements of the principal vector structure need to be restrained through complementary components. The moment balance is realized primarily based on the idea of symmetrically designing of the system, similar to adding a slider to accomplish moment balancing. The added components are designed to cancel out the static and dynamic forces and moments due to the motion of the mechanism. This moment balancing approach (symmetrically designing of the system) above in some extent is very similar to the approach of dynamic balancing through trajectory planning, as discussed in Section 3.8. Furthermore, the gripper studied in ref. [15] is actually originated from employing the principal vector linkage along with a slider. The principal vector linkage's motion is restricted so as to accomplish the moment balancing condition. Similarly in refs. [16-18], the authors employed the approach of Fisher's method; however, the moment balance in the work ${ }^{16-18}$ is achieved through appropriate choice of passive joints and specific trajectory path. Overall, the limitation in Wijk's work is that the method is only applicable to mechanisms that move within a single plane, and the method is not applicable to spatial mechanisms due to the complexity of the spatial kinematics. Also, the moment balancing technique is very restricted and it only applies to special cases.

Furthermore, a Dual-V type mechanism is developed from pantographs in ref. [19]. The shaking moment balancing of the overall system is mainly achieved by symmetrically placing four limbs, as shown in Fig. 2(c). The purpose of symmetrically designing the limbs in such way is that the shaking moment generated by each limb is in an opposite direction and thus the shaking moment can be balanced out each other. 
(a)
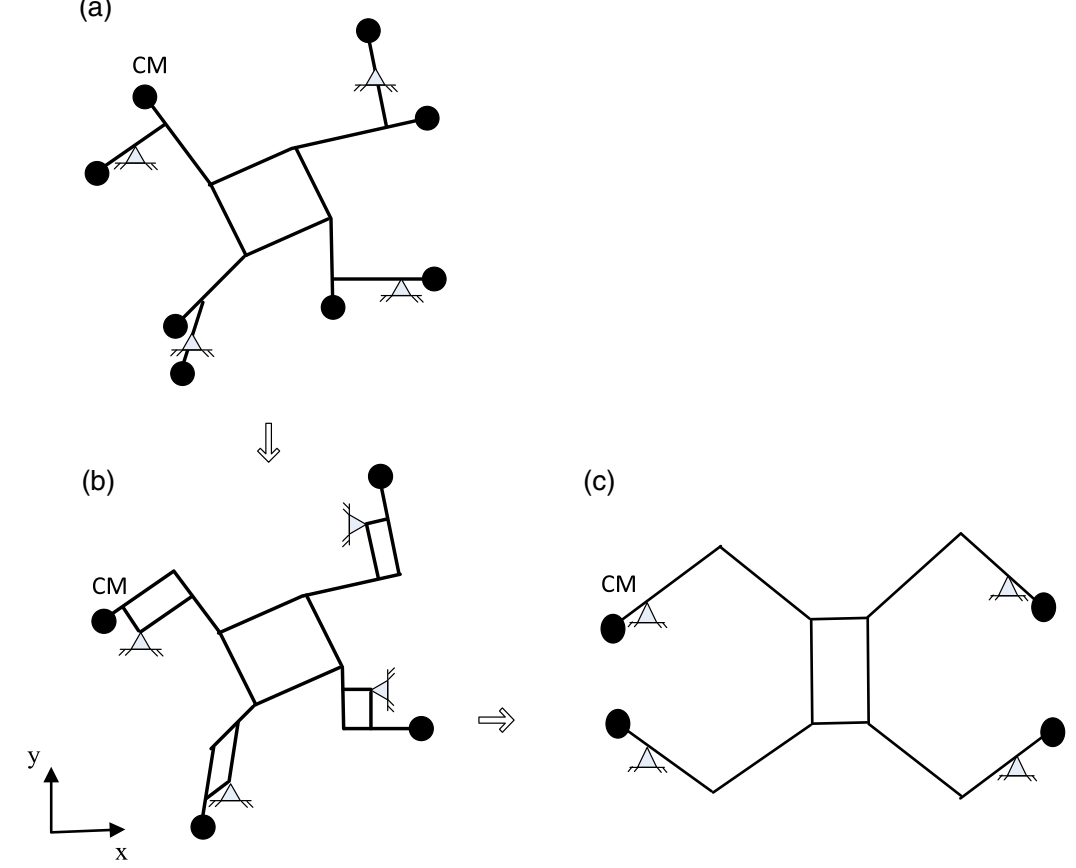

Fig. 3. Development of the 4-RRR balanced manipulator. ${ }^{20}$

The advantage of such design is that counter-rotations are not needed. However, the limitation of the above shaking moment balancing approach is that the robotic system is only dynamically balanced when the end-effector moves along the orthogonal lines (i.e., moves along $x$ and $y$ axes). When the robotic system moves along other directions rather than the $x$ and $y$ axes, it is not moment balanced anymore. As a side note, the developed Dual- $\mathrm{V}$ mechanism, from a different perspective, can also be designed from a normal planar mechanism with counterweights, as shown in Fig. 3(a), after which the four limbs are developed to parallelogram shaped structure, as shown in Fig. 3(b), and finally to the Dual-V mechanism. The counter-masses are represented by CM (i.e., the balancing components), as shown in Fig. 3.

\subsection{Using dynamically balanced four-bar linkage}

In the above, the authors incorporate methods of dynamic balancing within the kinematic synthesis of the mechanisms. This approach is designated in the introduction and herein this paper as "balancing prior to kinematic synthesis." This balancing-synthesis approach is also found in textbooks widely used for teaching undergraduate design of machinery. Another one that can also be considered as this technique is by using the dynamically balanced four-bar linkages, which is discussed in this section.

It is possible to use balanced 4-bar linkage to synthesize planar and spatial reactionless mechanisms. ${ }^{21-24}$ For example, in ref. [23], a 3-DOF mechanism is designed through combining two horizontal four bars and one vertical four bar. This mechanism is used as a building block (it is a reactionless mechanism, or it can be considered as a balancing component) to synthesize the 6-DOF parallel robot. Through calculation it is found that the four-bar linkage is not dynamically balanced when it is moving in three dimensions, and thus the 3-DOF mechanism structure is created. The overall robotic structure demonstrates that the reactionless conditions can be fulfilled. However, the downside of the above method is that the complexity of the overall system increases significantly, which complicates the kinematic and dynamic analysis.

Similarly, a four-bar structure (the so-called Gosselin type II reactionless mechanism), as shown in Fig. 5, is used to synthesize planar and spatial 3-DOF robots. ${ }^{24}$ The merit of this approach is that one does not add counter-rotations, but the platform is assumed to be very thin, which is not practical in reality. Very interestingly and from a different perspective, the four-bar can actually be viewed as the derivation of the principal vector chain. The three-chain linkage is advanced to a four-bar through attaching one linkage to the base, as illustrated in Fig. 4(b), and through reconfiguring and manipulating the moment balancing conditions and also the position of CoM of each link for the 
(a)

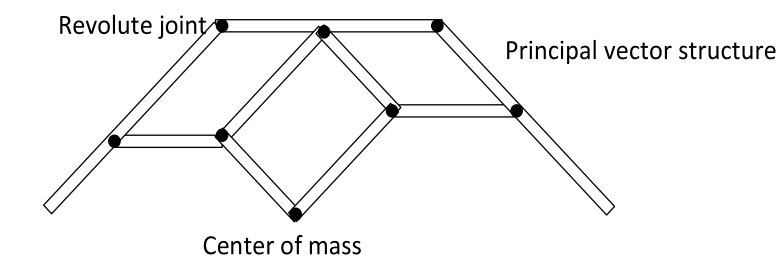

(b)
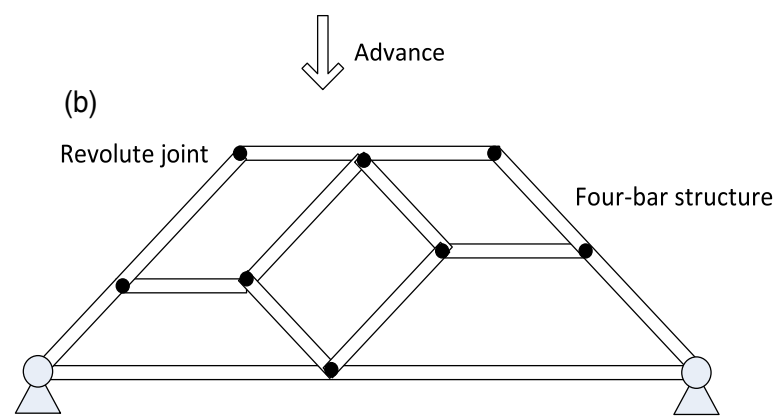

Fig. 4. Evolving process. ${ }^{20}$

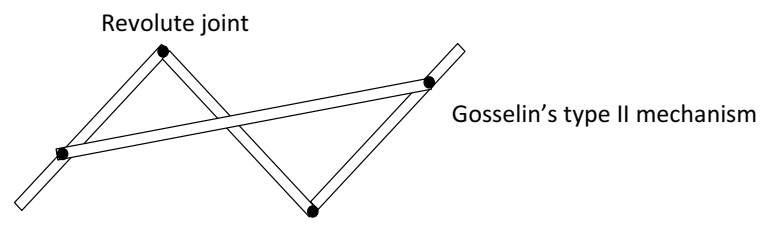

Fig. 5. Gosselin type II reactionless mechanism. ${ }^{21}$

four-bar structure (the detailed reconfiguring and derivation processes of the balancing conditions, i.e., how Fig. 5 is obtained from Fig. 4, are illustrated in ref. [21], the authors thus will not repeat it here anymore), the Gosselin type II reactionless mechanism can essentially be derived, as shown in Fig. 5. The points in Figs. 4 and 5 represent revolute joints.

\section{Balancing After the Design}

While two main dynamic balancing prior to kinematic synthesis methods are studied in Section 2, most of the dynamic balancing methods belong to the balancing after the design approach. Based on different techniques, the authors further divide the dynamic balancing methods into eight main groups: add separate counter-masses and counter-rotations method, add special four-bar linkage method, add springs method (only applies to static balancing), add CRCM method, add active-driven CRCM method, add active dynamic balancing unit method, add secondary linkages method, and balancing through trajectory planning and control method. The authors will discuss each of them in this section as follows.

\subsection{Add separate counter-masses and counter-rotations}

One can add counter-masses and counter-rotations to achieve a dynamic balancing condition. Regarding the shaking force balancing, the traditional way to make the CoM of the system fixed at a stationary point is to use counterweights to relocate the CoM to a fixed point. ${ }^{25-29}$ Regarding the shaking moment balancing, one can add counter-rotations to make the angular momentum equal to zero. ${ }^{30-36}$ For example, as shown in Fig. 6, one adds two counter-masses to a 2-DOF robotic manipulator to make the center of the mass of the system fixed at the base revolute joint; similarly, as shown in Fig. 7, one adds a counter-rotation to a rotating mechanism to achieve a shaking moment balancing condition.

A parallelogram five-bar-based structure is designed and being used as one limb for the planar three-DOF mechanism in ref. [38]. The mechanism has a pair of parallelogram five-bar linkage 


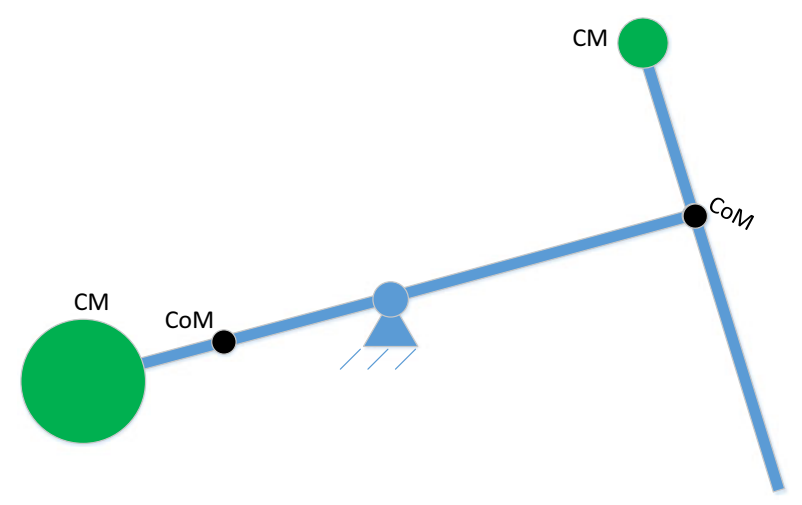

Fig. 6. Traditional counter-mass method for force balancing. ${ }^{37}$

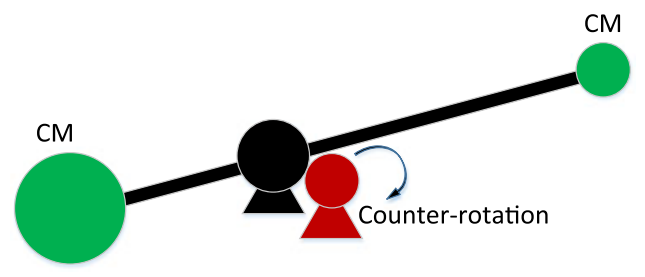

Fig. 7. Counter-rotation method for moment balancing. ${ }^{61}$

limbs. First of all, the moving platform was considered as two masses stationed at the attachment points between the limbs and the platform. To do that, the following requirements need to be fulfilled: one needs to have equivalent mass, equivalent inertia, and equivalent CoM. Then for every limb, the force balancing needs to be fulfilled at the beginning so as to attain the reactionless conditions. For the force balancing issue, the CoM formula $\mathrm{Mr}=\sum m_{i} r_{i}(r$ is the position of CoM) was applied. Through equaling the CoM position to zero, the force balancing solutions can be derived. According to the force balancing solutions, one can see that the mass and length terms are larger than zero. The exclusive approach to fulfill the condition is to let the CoM position of other linkages be smaller than zero. In order to fulfill this, counter-masses are employed. Regarding the moment balancing, the angular momentum of the five-bar system was determined, and through letting the angular momentum equal zero, moment balancing solutions can be obtained. From the force balancing, one establishes two equations, and based on the angular momentum conditions, one establishes three equations; that is, there are five equations being given in order to have the reactionless conditions for the limb. The originality of the above work is that the parallelogram structure was put forward to be used as one limb for a planar 3-DOF mechanism.

The above method is based on the concept of decomposing first and then integrating; that is, one limb is first proposed, making this limb achieve dynamic balanced conditions, and lastly those limbs are integrated to make up the entire mechanism. However, the drawback of the previous dynamic balanced system is that counter-devices were employed, which increases the weight, and the system was made more complex. The purposes of using counterweights and counter-rotations are to bring the CoM position to a fixed point and to keep the angular momentum a constant value, respectively.

In another study, ${ }^{39,40}$ the $\mathrm{CoM}$ of the system was first determined by using counter-masses; then through employing the linear momentum formula, the CoM was made still so as to satisfy the force balancing. Afterward, the shaking moment of the link can be modeled as the time change rate for the overall angular momentum. The model of the overall angular momentum of the linkage $H_{o}=\sum m_{i}\left(x_{i} \dot{y}_{i}-y_{i} \dot{x}_{i}+K_{i}^{2} \dot{\phi}_{i}\right)$ was used, after which the overall angular momentum is set at zero so as to obtain the reactionless conditions. Hereafter, it is found that one cannot achieve dynamic balancing unless counter-rotations are added. After counter-rotations were added to the system, the overall angular momentum was set at zero, and the moment balancing solutions were finally determined. 
However, the downside of this balancing approach is that the counter-balance device is placed on the moving links rather than placing it on the base, which requires us to counter-balance the effect generated by the counter-balance device.

A dyad achieves the reactionless condition through resorting to two counter-masses and two counter-rotations in ref. [41]. The counter-masses are put at each linkage, similar to the conventional force balancing approach, ${ }^{42}$ to have the CoM settled at the hinge. One accomplishes the shaking moment balancing through resorting to planetary gear trains that conduct the counter-rotations. The force balancing condition can be determined through resorting to the CoM equation. According to the force balancing solutions, one found that the terms of mass and length are larger than zero. The exclusive approach is to employ counter-masses. The upper movable linkage's shaking moment can be eliminated through one gearwheel, which is put on the ground. A small gear and a large gear on the ground are fixed side by side. The counter-rotation gearwheel on the left side is mated with the larger gearwheel, and the small gearwheel is attached to the top moveable linkage through a belt. Through this, the gearwheel is not directly joined to the top movable linkage, but it rotates contrary to the top movable linkage to have the reactionless conditions. With respect to the moment balancing, the angular momentum was first determined, and by equaling the angular momentum to zero, moment balancing conditions were extracted.

The drawback of the above dynamic balancing approach is that counter-masses and planetary gear trains are employed, which increases the overall weight and makes the system more complex. In the second part of the work, the shaking moment balancing was also investigated through resorting to flywheels and this solution is more promising. The angular momentum of the mechanism system was first obtained. For the purpose of realizing the moment balancing of the system, a flywheel can be employed. The flywheel has the equal and contrary shaking moment so that the system is moment balanced by a flywheel. One actuator drives the flywheel. This falls into the category of the active balancing approach. A similar study can also be found in ref. [43]. However, the issue of how to effectively and practically join the flywheel to the system still remains.

The scheme of mounting the gearwheel on the base, which is to counter balance the shaking moment, can result in a smaller increase of weight. ${ }^{44}$ The gearwheel is initially installed on the moving linkage, thus the counter-mass of the base linkage also needs to force balance this gear. If the gear is installed on the system frame, the base linkage counter-mass does not have to force balance this gearwheel anymore, which implies the mass for the counterweight of the base linkage could be reduced. However, the downside is that one increases the number of added components. ${ }^{42}$ The balancing scheme that the gearwheel installed on the system base is a derivative of Gao's approach in refs. $[39,40]$. A similar study can also be found in refs. [45, 46].

There is one study as follows employing nine counter-devices to achieve a reactionless condition. ${ }^{47}$ A 3-DOF parallelepiped structure is employed to synthesize the spatial robots. The parallelepiped structure has total of nine counter-devices (six counter-masses and three counterrotations) in order to make the robot to be dynamically balanced, which significantly increases the robot weight and inertia. This structure is not promising since one employed too many counter-masses and counter-rotations.

In ref. [37], the authors proposed the dynamic balancing through reconfiguration method to address the issue of using extra counter-devices. The basic concept of the dynamic balancing through reconfiguration approach is to balance the robotic system not through adding extra counter-devices, but rather through reconfiguring the robotic structure. One major advantage of which is that it can reduce the overall weight of the robotic system. One needs to firstly dynamically balance one limb through the reconfiguration approach and then unite the balanced limbs to design the overall robotic manipulator. The authors here will not reiterate it anymore. Details can be referred to ref. [37].

\subsection{Add special four-bar linkage}

Two types of Assur groups in ref. [48] are, respectively, used to dynamically balance a four-bar linkage without counter-rotations. The counterweights are used to force balance the four-bar linage, and the Assur group is used to moment balance the four-bar linkage. However, the structure of the overall system becomes complex, and also the added Assur group has the potential to collide with the four-bar linkage. It should be noticed that the difference between adding the four-bar linkage here and using balanced four-bar linkage in Section 2.2 is that in Section 2.2, the four-bar linkage is used to synthesize reactionless mechanisms before the mechanism is designed, whereas the adding 
four-bar linkage here is to add extra device to make the system to be dynamically balanced, in which the balancing condition is achieved after the mechanism being already designed.

Similarly, two methods are introduced to dynamically balance a planar robot mechanism in ref. [49]. One method is to add idler link between the base and the moving platform, but lots of counter-masses and counter-rotations are used, which greatly increases mass and inertia. The other approach is to add crank-slider mechanisms to all the mechanism limbs. This approach reduces the number of counter-rotations used. The second approach falls under the category of the passive dynamic balancing technique. If the passive balancing approach is changed to the active balancing approach, one can further cut down the number of counter-rotations. The counter-masses (i.e., the balancing components) are presented by $\mathrm{CM}$.

\subsection{Add springs}

There are several studies that focus on the static balancing of planar and spatial robotic mechanisms by incorporating springs into the robotic system. ${ }^{50-53}$ Following the previous studies in refs. [50-52], some recent studies ${ }^{54,55}$ formulated the force balancing of the robotic mechanism as a constrained nonlinear optimization problem, in which a mean-square root of the sum-squared values of bearing and spring forces is minimized, by using the "fmincon()" provided by the Matlab optimization toolbox. The above study combines the counter-masses and a spring that is attached between two passive links to achieve the force balancing condition. The limitation of the above method, however, is that it may only be applicable to planar mechanisms with revolute joints. The study did not prove whether the approach can apply to spatial robotic mechanisms.

Overall, the advantage of combining counter-masses and springs in force balancing is that the system will not be heavier as comparing to only using counter-masses, and also by employing a spring, it can make the robotic system adapt to different payloads and make the robotic system come to a new equilibrium configuration. ${ }^{56}$ However, the method proposed in this study is only applicable to shaking force balancing and ignores the shaking moment balancing. Other drawbacks of the above approach are summarized in ref. [57].

\subsection{Add CRCM}

The main idea of using a CRCM in dynamic balancing a mechanism is to use the same device to achieve both force balancing and moment balancing instead of using separate counter-masses and counter-rotations, which can reduce the mechanism's weight and inertia. ${ }^{58-60}$ However, the potential issue is that how to connect gears to the oscillating links without generating noise. In ref. [61], the main focus is on the "shift modification rule," from which the counter-rotary counterweight was developed, and it was compared to the technique that uses only counter-rotation. It was found that the CRCM approach achieved the goal of reducing extra masses and inertia. ${ }^{62}$ Similarly in ref. [63], the authors compared the following approaches for the amount of weight and inertia increase in balancing the dyad: counter-rotations approach, duplicate mechanisms approach, CRCM approach, and Idler loop approach. A judging element was set up. The objective of the element is to assess the extra weight and inertia increase. There are four main steps in this comparison, and here it is summarized as follows:

First step: The counterweights and lump mass's position vectors were determined, then by employing the position vector derivatives, the linear momentum was extracted. By making it zero, the force balancing conditions were obtained.

Second step: The angular momentum with respect to the reference point was extracted. The relationship between the gearwheels was used to further simplify the momentum, and through making it equal to zero, the reactionless conditions were therefore obtained.

Third step: This involves determining the reduced inertia through the kinetic energy.

Fourth step: The overall mass is obtained.

Last step: The overall mass and inertia reduction to the previous approaches are compared.

However, it should be noticed that it is unnecessary to make a comparison for the mass and inertia reduction due to the fact that some of the masses and inertia are on the base, so the weight that belongs to the base does not directly have an influence on the system. 


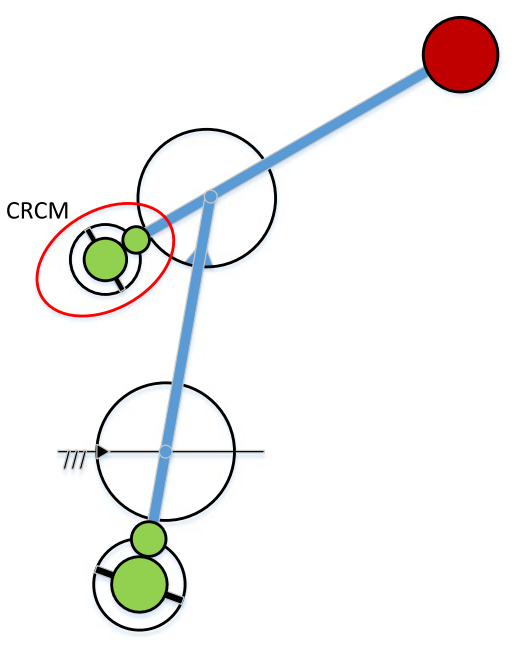

Fig. 8. Single CRCM structure. ${ }^{64}$

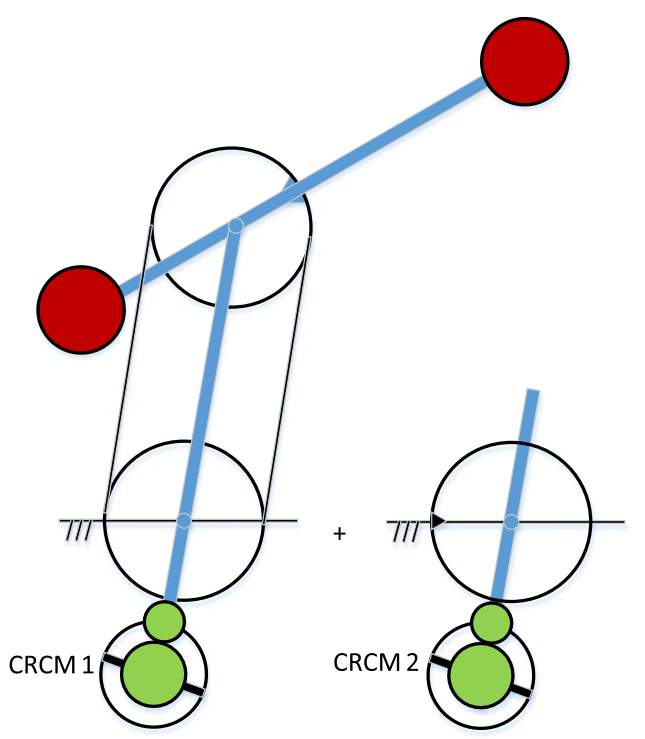

Fig. 9. Ground CRCM balancing approach. ${ }^{64}$

The following three CRCM-based methods are developed in ref. [64]: the low inertia approach, single CRCM approach, and ground CRCM balancing approach. Each approach has its advantages. For example, the low inertia approach has low inertia increase, the CRCM approach only requires one CRCM for dynamic balancing, and the ground CRCM balancing approach has compact construction. Lastly, numerous 2-DOF manipulators were designed based on the CRCM structure. Moreover, the 3-DOF planar and spatial mechanisms were designed through resorting to the reactionless dyad.

It should be noticed that the single CRCM structure is not recommended because the balancing gears (as indicated by the red circle of Fig. 8) are placed on the moving linkage rather than on the ground. The ground CRCM balancing approach is similar to the Idler loop, ${ }^{41}$ that is, the moment of the top movable linkage is balanced through a CRCM, which is joined to the top movable linkage by a belt drive, as shown in Fig. 9. The moment of ground linkage is balanced through a second CRCM, which is joined to a gearwheel, and this gearwheel is installed on the ground linkage, which makes the mechanism heavier. As a comparison, the structure in refs. [41,44] is that the gear, which moment balances the top movable linkage, is put on the ground, so the system is not affected at all.

By using the similar idea, a reactionless 2-DOF planer parallel mechanism is developed by using the same counter-mechanism to moment balance the statically balanced mechanism, ${ }^{65,66}$ instead of 


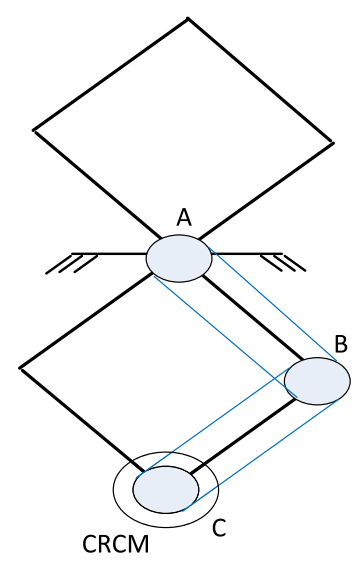

Fig. 10. AD-CRCM-balanced mechanism. ${ }^{67}$

using counter-rotations. The counter-mechanism achieves variable inertia, reduces the mass of the reactionless mechanism that uses counter-rotations, and has better mass-inertia performance, but still the additional mass and inertia are added in the system, which makes the system become heavier.

\subsection{Add active-driven CRCM}

A dyad is dynamically balanced by actively driving the CRCM (AD-CRCM) in ref. [67]. The rotational motion velocity of the AD-CRCM and the actuator torque that drives the CRCM are determined from the angular momentum. Via comparison, it is found that the AD-CRCM method is better than the normal CRCM method in terms of weight and inertia relations. By combining two CRCM into one AD-CRCM, a 2-DOF reactionless structure can be generated, as illustrated in Fig. 10. The 3-DOF planar and spatial mechanisms are designed through resorting to the AD-CRCM reactionless dyad. Since the AD-CRCM was employed, the system becomes heavier.

In the above study, a 2-DOF AD-CRCM balanced mechanism was designed through joining two CRCM to one AD-CRCM. Enlightened from the previous layout, new 3-DOF planar and spatial mechanisms can be designed, as illustrated in Fig. 11, based on using the 2-DOF AD-CRCM reactionless structure. Note that this is just a conceptual design. Since the kinematic, dynamic, and control analysis of these 3-DOF planar and spatial mechanisms are not the focus of this study, they will be studied in a separate paper as a future work.

\subsection{Active dynamic balancing unit}

An ADBB is introduced in ref. [68], and it is placed on the base of the system. The purpose of using this ADBB is to produce same counter forces and counter moments but have opposite direction with the existing shaking forces and moments of the system. A similar idea can also be found in ref. [69]. The ADBB has three counterweights and three counter-rotations. The objective of the three counterweights is to force balance the pre-existing shaking force in three directions. The objective of the three counter-rotations is to moment balance the shaking moment in three directions. This approach is similar to the adding separate counter-masses and counter-rotations method. Considering the low mass inclusion, the ADBB is advanced to another ADBB so that the counter-devices are united. Here, the system has the shaking forces along the $x$ and $y$ axes and one shaking moment around the $z$ axis. Since the ADBB has to make two pre-existing shaking forces and one pre-existing shaking moment be balanced, the ADBB is developed to have two translational movements and one rotational movement. A 2-RRR parallel manipulator was employed to actuate the disc in the $x$ and $y$ axes. Potential future study is to seek the intelligent control approach for manipulating the ADBB. However, one issue in this study is that it will make the system become heavier if the base is suspended.

Wang $^{70}$ suggested an active reactionless structure almost identical to the ADBB as in ref. [68]. By using this active reactionless structure, the moving platform can achieve both reactionless conditions and can be actuated to proceed in a designated path. The primary objective of the active reactionless structure is to make the moving platform to have the reactionless condition. A portion of the forces 
(a)

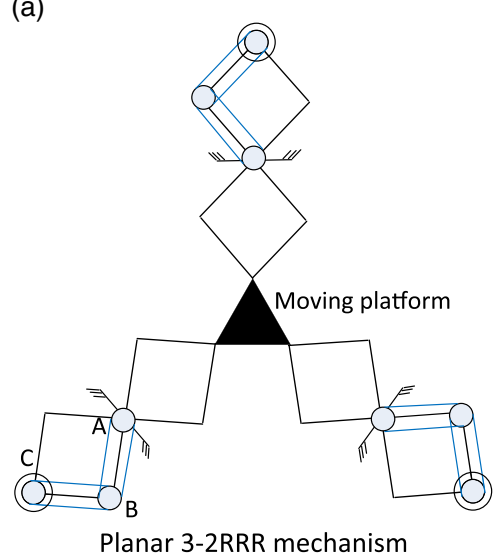

(c)

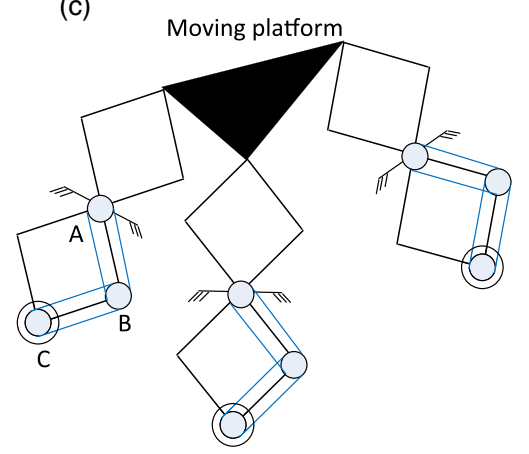

Spatial 3-2RRR mechanism (b)
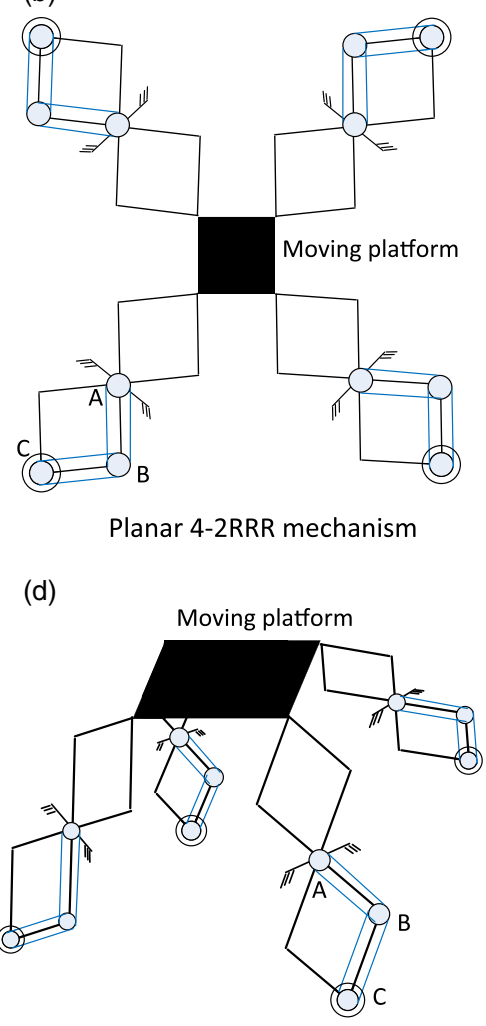

Spatial 4-2RRR mechanism

Fig. 11. 3DOF mechanisms. ${ }^{20}$ (a) Planar 3-2RRR mechanism. (b) Planar 4-2RRR mechanism. (c) Spatial 32RRR mechanism. (d) Spatial 4-2RRR mechanism.

and moments generated through the active reactionless structure is employed to make the shaking force and moment be balanced. The rest of the portion of the forces and moments generated through the active reactionless structure are employed to drive the platform to a designated path. One of the main issues is the same as above that the system becomes heavier.

\subsection{Add secondary linkages}

Regarding achieving the reactionless conditions for the Delta manipulator in ref. [71], an approach was suggested for the force balance. All limbs and one-third of the platform are balanced by a counterweight and one extra linkage; that is, each leg turns into a 3D-pantograph. This approach is in some extent very similar to the method of adding counter-masses and springs together, as discussed in Section 3.3, where counter-masses and springs are added together to achieve the force balancing. Moreover, because the platform of the Delta system does not have rotational motion, the force balancing approach was reduced as: one limb as a 3D-pantograph balances the total weight of the platform and part of the weight of the other two limbs. Two extra counterweights are put on the other two limbs. The complete manipulator is made to be force balanced through three counterweights and an extra linkage. Regarding the moment balancing, the active-driven method was employed because one would not be able to let the velocity of the manipulator be a constant value through the passive balancing approach. It was discovered that the weight of the extra linkage can be employed to force balance the total mass of the platform and the upper linkages (which are close to the platform). However, the downside of this balancing approach is that still the system's weight and inertia will increase.

\subsection{Balancing through trajectory planning and control}

There are some studies dealing with optimum motion planning to achieve a dynamic balancing condition. ${ }^{72-75}$ More precisely, most studies above still use counter-devices to achieve a force balancing 
condition, and after that by optimal control of the end-effector to achieve a shaking moment balancing condition. For example, in ref. [73], the SCARA robot is dynamically balanced through using counter-masses and optimal control. The purpose of the counter-mass is to balance the shaking force, and through controlling the serial wrist with prescribed rotation velocities, shaking moment is cancelled. The novelty of this balancing approach is that an optimal control is employed to achieve the moment balancing.

In another study, the reactionless planar mechanisms design is presented through trajectory planning instead of using counter-rotations, ${ }^{74}$ which reduce the system weight and inertia. An auxiliary parallelogram is used to achieve the center of the mass of the system stationary (i.e., to achieve force balancing), and the moment balancing is achieved through following a special trajectory. Similarly in ref. [75], through a specific reactionless trajectory planning, a moment balancing condition of a 3-DOF robotic manipulator is achieved instead of using counter-rotations.

As one can imagine, the major disadvantage of the above approach is that it limits the end-effector trajectory. Another study ${ }^{76}$ which is worth mentioning is that through adjusting kinematic parameters and therefore the end-effector trajectories, the force balancing condition is achieved, but it does not apply to the dynamic balancing.

In addition, there are several studies formulating the dynamic balancing as a constrained optimization problem to try to eliminate the shaking force and shaking moment of mechanisms by increasing the applied torques of some driving joints instead of using counter-devices, ${ }^{77-83}$ but it should be noted that most optimization studies above are based on numerical methods.

\section{Comparisons}

Here, some advantages, disadvantages, and comparisons of each balancing method are listed in Table I. Each method has its advantages and disadvantages. It is hard to say at this point that what the best approach to dynamic balancing is. Depending on different applications and users' requirements, one may choose one balancing method over the other.

\section{Impact of Dynamic Balancing and Future Direction}

Dynamic balancing is critical in many areas, ${ }^{84-88}$ such as in advanced manufacturing and space. In manufacturing, for example, if a robotic system/mechanism used in machining is not dynamically balanced, it will excite vibrations and therefore cause loss of high precision in production of workpieces. Furthermore, if the robotic system is not dynamically balanced, it will create vibration and one therefore needs a large stand or frame to hold the robotic system/mechanism, which will occupy large space in the (manufacturing) floor, as can be seen in Fig. 12, and also create internal force. If the robotic system is perfectly dynamically balanced, one can use, for example, a small frame or simply a rod to hold the robotic system without producing vibration. Similarly in space engineering, for example, as shown in Fig. 13, when robotic arms operate in space and if they are not dynamically balanced, the robotic mechanism will generate force and moment in the base (spacecraft), which affects the performance of the spacecraft.

There are mainly two methods that belong to the dynamic balancing prior to structure synthesis, while most of the balancing approaches belong to the dynamic balancing after the design. As mentioned earlier, in an absolute sense, it is hard to say which method/approach is definitely better than the other or what the best approach to dynamic balancing is. Each method has its advantages and disadvantages. For example, the drawback of using counterweights approach is that the system will become heavier; the drawback of using trajectory planning method is that the system will only have a reactionless condition in a specific trajectory. The limitation of the principal vector linkage approach is that it is only applicable to mechanisms that move within a single plane. Adding springs method is currently only applicable to static balancing. The potential issue of using CRCM approach is how to connect gears to the oscillating links without generating noise.

As regarding the future trend and direction in the dynamic balancing of robotic mechanisms, it will be interesting to investigate the dynamic balancing through adaptive control technique, and this could potentially be an effective approach without compromising system's weight, inertia, trajectory, or complexity, since the main idea behind the adaptive control is that if one has unknown parameters in a dynamic system, through adaptive control we can make the system behave as if the unknown parameters were known by updating the unknown parameters. Furthermore, dynamic balancing for spatial mechanisms still needs to be more generalized, since most dynamic balancing 
Method (balancing prior to

kinematic synthesis)

Advantages

Disadvantages

Fisher's method (representative: Wijk's work)

Using dynamically balanced four-bar linkage*
- Counter-rotations are not needed

- Overall weight is reduced as compared to adding separate

counter-rotations method

- Counter-rotations are not needed

- Overall weight is reduced as compared to adding separate counter-rotations method
- Not applicable to spatial mechanisms due to the complexity of the spatial kinematics

- Moment balance is realized based on adding extra components

- Complexity of overall system increases significantly

- Kinematic and dynamic analyses are complicated

Method (balancing after design)

Add separate counter-masses and

counter-rotations

Add special four-bar linkage*

Add springs

Add CRCM

Add active driven CRCM

Add active dynamic balancing unit

Add secondary linkages

Balancing through trajectory planning and control
- Overall weight is increased significantly

- The number of counter-rotations are reduced or sometimes no needed

- System weight is reduced as compared to only using

counter-masses

- Counter-mass is also used as counter-rotation

- System weight and inertia are reduced as compared to adding separate counter-masses and counter-rotations

- Active-driven CRCM method is better than the normal CRCM method in terms of weight and inertia relation

- Both counterweights and counter-rotations are needed, similar

to separate counter-masses and counter-rotations method

- System weight (the number of counter-masses) is reduced as compared to only using counter-masses

- Counter-rotations are not needed

- System weight and inertia are reduced
- Simple approach to achieve dynamic balancing

Structure of overall system is complex

- Added linkage has the potential to collide with others

- Only applicable to static balancing

- Potential issue is how to connect gears to oscillating links without generating noise

- Counter-devices still used

- System becomes complex

- Difficult to realize in practice

- System is heavier as compared to CRCM approach

- System becomes heavier

- Only for force balancing

- Active-driven method is needed to moment balancing

- System weight and inertia are increased

- End-effector trajectory is limited

* Noted that the main difference between adding the four-bar linkage and using the dynamically balanced four-bar linkage is that the dynamically balanced four-bar linkage is used to synthesize reactionless mechanisms before the mechanism is designed, whereas the adding special four-bar linkage is to add extra device to make the system to be dynamically balanced, in which the balancing condition is achieved after the mechanism being already designed. 


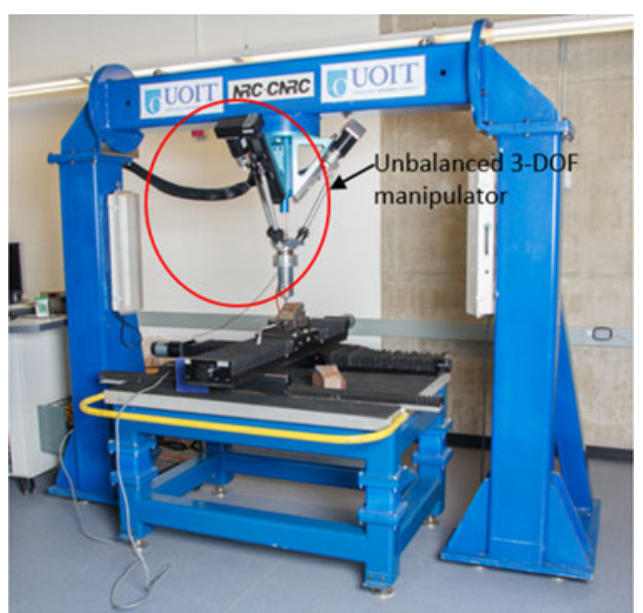

Fig. 12. 3-DOF robotic manipulator and its stand.

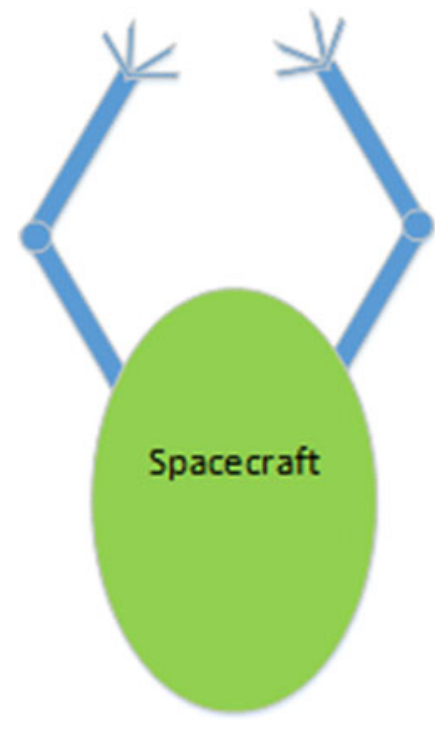

Fig. 13. Robotic arms attached to a spacecraft.

methods for spatial mechanisms all have constrains due to the complexity of the spatial kinematics and its structure.

\section{Conclusion}

The authors here divide dynamic balancing methodology into two main categories: balancing prior to structure synthesis and balancing after the design, and the main dynamic balancing methods for robotic mechanisms based on these two categories are discussed and compared. There could be other methods that the authors did not include in this study. The purpose of dynamic balancing is to reduce or ideally eliminate the reaction forces and reaction moments exert to the system, which causes vibration. The drawback of achieving a dynamic balancing, however, is mainly as follows: weight increase, inertia increase, trajectory limitations, and adding complexity to the system.

The purpose of this review study is to present the up-to-date main dynamic balancing methods. The authors hope that this review can provide an informative reference for future research in the field of dynamic balancing of robotic mechanisms.

\section{Acknowledgments}

The authors would like to thank the financial support from the Natural Sciences and Engineering Research Council of Canada (NSERC). The authors would also like to acknowledge the financial support from the Kaneff Research Chairs program at York University. 


\section{References}

1. R. S. Berkof and G. G. Lowen, "A new method for completely force balancing simple linkages," J. Eng. Ind. 91(B), 21-26 (1969).

2. R. S. Berkof, "Complete force and moment balancing of inline four-bar linkages," Mech. Mach Theory 8(3), 397-410 (1973).

3. G. G. Lowen and R. S. Berkof, "Survey of investigations into the balancing of linkages," J. Mech. 3(4), 221-231 (1968).

4. R. Ricard and C. M. Gosselin, "On the Development of Reactionless Parallel Manipulators," Proceedings of ASME Design Engineering Technical Conferences and Computers and Information in Engineering Conference, USA (2000) pp. 1-10.

5. K. Yoshida, K. Hashizume and S. Abiko, "Zero Reaction Maneuver: Flight Validation with ETS-VII Space Robot and Extension to Kinematically Redundant Arm," Proceedings of IEEE International Conference on Robotics and Automation (2001) pp. 441-446.

6. F. Xi and R. Sinatra, "Effect of dynamic balancing on four-bar linkage vibrations," Mech. Mach. Theory 32(6), 715-728 (1997).

7. F. Xi and Z. Qin, "An integrated approach for design and analysis of a fluid mixer," Comput. Aided Des. 30(13), 1037-1045 (1998).

8. S. D. Yu and F. Xi, "Effect of balancing on dynamic instability of high-speed flexible four-bar mechanisms," CSME Trans. 26(2), 139-162 (2002).

9. C. Gosselin, B. Moore and J. Schicho, "Dynamic balancing of planar mechanisms using toric geometry," J. Symb. Comput. 44(9), 1346-1358 (2009).

10. C. Gosselin, "Gravity Compensation, Static Balancing and Dynamic Balancing of Parallel Mechanisms," In: Smart Devices and Machines for Advanced Manufacturing (L. Wang and J. Xi, eds.) (Springer, London, 2008) pp. 27-48.

11. C. Gosselin, "Static balancing of spherical 3-DOF parallel mechanisms and manipulators," Int. J. Robot. Res. 18(8), 819-829 (1999).

12. J. Herder and V. Wijk, Force balanced delta robot. Patent NL2002839 (2010).

13. D. Zhang and B. Wei, "Discussion and Analysis of Main Dynamic Balancing Methods for Robotic Manipulators," Proceedings of ASME 2016 International Design Engineering Technical Conferences \& Computers and Information in Engineering Conference, Charlotte, USA (2016) Paper no: V006T09A009, $7 \mathrm{p}$.

14. O. Fischer, Theoretische grundlagen für eine Mechanik der lebenden Körper (Teubner, Leipzig, 1906).

15. V. Wijk, Methodology for Analysis and Synthesis of Inherently Force and Moment-Balanced Mechanisms - Theory and Applications, Ph.D. Dissertation (University of Twente, Netherlands, 2004).

16. A. Fattah and S. K. Agrawal, "Design and Modeling of Classes of Spatial Reactionless Manipulators," Proceedings of IEEE International Conference on Robotics and Automation (2003) pp. 3225-3230.

17. A. Fattah and S. K. Agrawal, "Design and simulation of a class of spatial reactionless manipulators," Robotica 23(1), 75-81 (2005).

18. S. K. Agrawal and A. Fattah, "Reactionless space and ground robots: Novel designs and concept studies," Mech. Mach. Theory 39(1), 25-40 (2004).

19. V. Wijk, S. Krut, F. Pierrot and J. L. Herder, "Design and experimental evaluation of a dynamically balanced redundant planar 4-RRR parallel manipulator,” Int. J. Robot. Res. 32(6), 744-759 (2013).

20. D. Zhang and B. Wei, "Review of Recent Advances on Reactionless Mechanisms and Parallel Robots," In: Dynamic Balancing of Mechanisms and Synthesizing of Parallel Robots (D. Zhang and B. Wei, eds.) (Springer, Cham, 2016) pp. 1-19.

21. Y. Wu and C. Gosselin, "Synthesis of reactionless spatial 3-DOF and 6-DOF mechanisms without separate counter-rotations," Int. J. Robot. Res. 23(6), 625-642 (2004).

22. A. Lecours and C. Gosselin, "Reactionless two-degree-of-freedom planar parallel mechanisms with variable payload," ASME J. Mech. Robot. 2(4), 041010 (2010).

23. Y. Wu, Synthesis and Analysis of Reactionless Spatial Parallel Mechanisms, Ph.D. Dissertation (Laval University, 2003).

24. C. Gosselin, F. Vollmer, G. Cote and Y. Wu, "Synthesis and design of reactionless three-degree of freedom parallel mechanisms," IEEE Trans. Robot. Autom. 20(2), 191-199 (2004).

25. V. Filaretov and M. Vukobratovic, "Static balancing and dynamic decoupling of the motion of manipulation robots," Mechatronics 3(6), 767-782 (1993).

26. M. Walker and K. Oldham, "A general theory of force balancing using counterweights," Mecha. Mach. Theory 13(2), 175-185 (1978).

27. K. Oldham and M. Walker, "A procedure for force-balancing planar linkages using counterweights," J. Mecha. Eng. Sci. 20(4), 177-182 (1978).

28. F. Xi, "Dynamic balancing of hexapods for high-speed applications," Robotica 17(3), 335-342 (1999).

29. H. Chaudhary and S. Saha, "An optimization technique for the balancing of spatial mechanisms," Mecha. Mach. Theory 43, 506-522 (2008).

30. C. Bagci, "Complete shaking force and shaking moment balancing of link mechanisms using balancing idler loops," J. Mech. Des. 104, 482-493 (1982).

31. L. Berestov, Full Dynamic Balancing of Pinned Four-Bar Linkage. Izvestiya Vysshikh Uchebnykh Zavedenii. Series: Machinostroenie, vol. 11 (1975), pp. 62-65. 
32. C. Tah, L. Yong and V. Alves, "Decoupling of dynamic equations by means of adaptive balancing of 2-dof open-loop mechanisms," Mecha. Mach. Theory 39(8), 871-881 (2004).

33. M. Acevedo, M. Ceccarelli and G. Carbone, "Application of counter-rotary counterweights to the dynamic balancing of a spatial parallel manipulator," Appl. Mech. Mater. 162, 224-233 (2012).

34. V. Arakelian and S. Sargsyan, "On the design of serial manipulators with decoupled dynamics," Mechatronics 22(6), 904-909 (2012).

35. I. Kochev, "General theory of complete shaking moment balancing of planar linkages: A critical review," Mecha. Mach. Theory 35(11), 1501-1514 (2000).

36. B. Moore, J. Schicho and C. Gosselin, "Determination of the complete set of shaking force and shaking moment balanced planar four-bar linkages," Mecha. Mach. Theory 44(7), 1338-1347 (2009).

37. D. Zhang and B. Wei, "Dynamic Balancing of Parallel Manipulators through Reconfiguration," Proceedings of ASME 2015 Dynamic Systems and Control Conference (2015) pp. 1-9, Paper No: V003T43A001, doi: 10.1115/DSCC2015-9669.

38. S. Foucault and C. Gosselin, "Synthesis, design, and prototyping of a planar three degree-of-freedom reactionless parallel mechanism," ASME J. Mech. Des. 126(6), 992-999 (2005).

39. F. Gao, "Complete shaking force and shaking moment balancing of 26 types of four-, five- and six-bar linkages with prismatic pairs," Mech. Mach. Theory 25(2), 183-192 (1990).

40. F. Gao, "Complete shaking force and shaking moment balancing of 17 types of eight-bar linkages only with revolute pairs," Mech. Mach. Theory 26(2), 197-206 (1991).

41. V. H. Arakelian and M. R. Smith, "Design of planar 3-DOF 3-RRR reactionless parallel manipulators," Mechatronics 18(10), 601-606 (2008).

42. D. Zhang and B. Wei, "Synthesizing of reactionless flexible mechanisms for space applications," Int. J. Space Sci. Eng. 5(1), 1-15 (2018).

43. V. Arakelian, S. Briot, S. Yatsun, et al., "A New 3-DoF Planar Parallel Manipulator with Unlimited Rotation Capability," Proceedings of the 13th World Congress in Mechanism and Machine Science, Mexico (2011) pp. $1-8$.

44. V. Arakelian and M. Smith, "Complete shaking force and shaking moment balancing of linkages," Mech. Mach. Theory 34(8), 1141-1153 (1999).

45. P. Nehemiah, "Complete shaking force and shaking moment balancing of 3 types of four-bar linkages" Int. J. Curr. Eng. Tech. 4(6), 275-287 (2014).

46. P. Nehemiah, B. Rao and K. Ramji, "Shaking force and shaking moment balancing of planar mechanisms with high degree of complexity," Jordan J. Mech. Ind. Eng. 6(1), 17-24 (2012).

47. Y. N. Wu and C. Gosselin, "Design of reactionless 3-DOF and 6-DOF parallel manipulators using parallelepiped mechanisms," IEEE Trans. Robot. 21(5), 821-833 (2005).

48. S. Briot and V. Arakelian, "Complete shaking force and shaking moment balancing of in-line four bar linkages by adding a class-two RRR or RRP Assur group," Mech. Mach. Theory 57, 13-26 (2012).

49. S. Briot, I. A. Bonev, C. M. Gosselin and V. Arakelian, "Complete shaking force and shaking moment balancing of planar parallel manipulators with prismatic pairs," Proc. Inst. Mech. Eng. Part K 223(1), 43-52 (2009).

50. J. Wang and C. Gosselin, "Static balancing of spatial three-degree-of-freedom parallel mechanisms," Mech. Mach. Theory 34(3), 437-452 (1999).

51. T. Laliberte, C. Gosselin and M. Jean, "Static balancing of 3-DOF planar parallel mechanism," IEEE/ASME Trans. Mech. 4(4), 363-377 (1999).

52. I. Ebert-Uphoff, C. Gosselin and T. Laliberteí, "Static balancing of spatial platform mechanism-revisit," J. Mech. Des. 122(1), 43-51 (2000).

53. A. Russo, R. Sinatra and F. F. Xi, "Static balancing of parallel robots," Mech. Mach. Theory 40(2), 191-202 (2005).

54. G. Alici and B. Shirinzadeh, "Optimum Force Balancing with Mass Distribution and a Single Elastic Element for a Five-bar Parallel Manipulator," 2003 IEEE International Conference on Robotics and Automation (2003), pp. 3666-3671.

55. G. Alici and B. Shirinzadeh, "Optimum force balancing of a planar parallel manipulator," Proc. Inst. Mech. Eng. Part C J. Mech. Eng. Sci. 217(5), 515-524 (2003).

56. D. A. Streit and B. J. Gilmore, "Perfect spring equilibrators for rotatable bodies," J. Mech. Transm. Autom. Des. 111(4) (1989). doi:10.1115/1.3259020.

57. V. Arakelian, "Shaking force and shaking moment balancing in robotics: A critical review," Mech. Mach. Sci. 22, 149-157 (2014).

58. V. Wijk and J. Herder, "Double Pendulum Balanced by Counter-Rotary Counter-Masses as Useful Element for Synthesis of Dynamically Balanced Mechanisms," Proceedings of the ASME International Design Engineering Technical Conferences \& Computers and Information in Engineering Conference, Brooklyn, NY (2008) pp. 453-463.

59. V. Wijk and J. Herder, "Guidelines for Low Mass and Low Inertia Dynamic Balancing of Mechanisms and Robotics," In: Advances in Robotics Research (T. Kröger and F. M. Wahl, eds) (Springer, Berlin, Heidelberg, 2009) pp. 21-30.

60. V. Wijk, B. Demeulenaere, C. Gosselin, et al., "Comparative analysis for low-mass and low-inertia dynamic balancing of mechanisms," ASME J. Mech. Robot. 4 (2012). doi:10.1115/1.4006744.

61. J. Herder and C. Gosselin, "A Counter-Rotary Counterweight (CRCM) for Light-Weight Dynamic Balancing," Proceedings of DETC 2004 ASME Design Engineering Technical Conferences and Computers and Information in Engineering Conference, USA (2004) pp. 1-9. 
62. J. Herder, Reaction-Free Systems. Principles, Conception and Design of Dynamically Balanced Mechanisms, Technical Report (Laval University, 2003).

63. V. Wijk, B. Demeulenaere and J. Herder, "Comparison of various dynamic balancing principles regarding additional mass and additional inertia," ASME J. Mech. Robot. 1(4), 041006-1-9 (2009).

64. V. Wijk and J. Herder, "Synthesis of dynamically balanced mechanisms by using counter-rotary countermass balanced double pendula," ASME J. Mech. Des. 131(11), 111003-1-8 (2009).

65. T. Laliberte and C. Gosselin, "Dynamic Balancing of Two-DOF Parallel Mechanisms using a CounterMechanism," Proceedings of ASME 2013 International Design Engineering Technical Conferences and Computers and Information in Engineering Conference, Portland (2013). doi:10.1115/DETC2013-12107.

66. T. Laliberte and C. Gosselin, "Synthesis, optimization and experimental validation of reactionless two-DOF parallel mechanisms using counter-mechanisms," Meccanica 51, 3211-3225 (2016). doi:10.1007/s11012-016-0582-0.

67. V. Wijk and J. Herder, "Dynamic Balancing of Mechanisms by using an Actively Driven Counter-Rotary Counter-Mass for Low Mass and Low Inertia," Proceedings of the Second International Workshop on Fundamental Issues and Future Research Directions for Parallel Mechanisms and Manipulators, France (2008) pp. 241-251.

68. V. Wijk and J. Herder, "Active Dynamic Balancing Unit for Controlled Shaking Force and Shaking Moment Balancing," Proceedings of the ASME Design Engineering Technical Conference (2010) pp. 1515-1522.

69. H. Dresig and N. Dien, "Complete shaking force and shaking moment balancing of mechanisms using a moving rigid body," Technische Mechanik 31(2), 121-131 (2011).

70. K. Wang, M. Luo and T. Mei, "Dynamics analysis of a three-DOF planar serial-parallel mechanism for active dynamic balancing with respect to a given trajectory," Int. J. Adv. Robot. Syst. 10(23), 1-10 (2013).

71. V. Wijk and J. Herder, "Dynamic Balancing of Clavel's Delta Robot," In: Computational Kinematics (A. Kecskeméthy and A. Müller, eds) (Springer, Berlin, Heidelberg, 2009) pp. 315-322.

72. S. Briot and V. Arakelian, "Complete Shaking Force and Shaking Moment Balancing of the PositionOrientation Decoupled PAMINSA Manipulator," Proceedings of the IEEE/ASME International Conference on Advanced Intelligent Mechatronics, Singapore (2009) pp. 1521-1526.

73. V. Arakelian and S. Briot, "Dynamic Balancing of the SCARA Robot," The 17-th CISM-IFToMM Symposium on Robot Design, Dynamics and Control (RoManSy 2008), Tokyo, Japan (2008) pp. 167-174.

74. A. Fattah and S. Agrawal, "On the design of reactionless 3-DOF planar parallel mechanisms," Mech. Mach. Theory 21(1), 70-82 (2006).

75. E. Papadopoulos and A. Abu-Abed, "Design and Motion Planning for a Zero-Reaction Manipulator," Proceedings of the 1994 IEEE International Conference on Robotics and Automation (1994) pp. 1554-1559.

76. P. R. Ouyang and W. J. Zhang, "Force balancing of robotic mechanisms based on adjustment of kinematic parameters," J. Mech. Des. 127, 433-440 (2005).

77. G. Alici and B. Shirinzadeh, "Optimum Dynamic Balancing of Planar Parallel Manipulators," Proceedings of the IEEE International Conference on Robotics and Automation, New Orleans (2004) pp. 4527-4531.

78. G. Alici and B. Shirinzadeh, "Optimum dynamic balancing of planar parallel manipulators based on sensitivity analysis," Mech. Mach. Theory 41, 1520-1532 (2006).

79. D. Ilia, A. Cammarata and R. Sinatra, "A Novel Formulation of the Dynamic Balancing of Five-Bar Linkages," Proceedings of the 12th World Congress in Mechanism and Machine Science, France (2007) pp. 193-211.

80. D. Ilia and R. Sinatra, "A novel formulation of the dynamic balancing of five-bar linkages with applications to link optimization," Multibody Syst. Dyn. 21, 193-211 (2009).

81. A. Buganza and M. Acevedo, "Dynamic Balancing of a 2-DOF 2RR Planar Parallel Manipulator by Optimization," 13th World Congress in Mechanism and Machine Science, Guanajuato, Mexico (2011) pp. $19-25$.

82. H. Chaudhary and S. K. Saha, "Balancing of four-bar linkages using maximum recursive dynamic algorithm," Mech. Mach. Theory 42(2), 216-232 (2007)

83. K. Chaudhary and H. Chaudhary, "Dynamic balancing of planar mechanisms using genetic algorithm," $J$. Mech. Sci. Tech. 28(10), 4213-4220 (2014).

84. Z. M. Bi and L. H. Wang, "Optimal design of reconfigurable parallel machining systems," Robot. Comput. Integr. Manuf. 25(6), 951-961 (2009).

85. M. Rosen and H.A. Kishawy, "Sustainable manufacturing and design: Concepts, practices and needs," Sustainability 4(2), 154-174 (2012).

86. Q. K. Liang, D. Zhang, Q. Song and Y. Ge, "Design and Evaluation of a Novel Flexible Bio-Robotic Foot/Ankle Based on Parallel Kinematic Mechanism," Proceedings of the 2010 IEEE International Conference on Mechatronics and Automation, Xi' an, China (2010) pp. 1548-1552.

87. W. Dong, Z. Du, Y. Xiao and X. Chen, "Development of a parallel kinematic motion simulator platform," Mechatronics 23(1), 154-161 (2013).

88. B. Zi, G. Yin and D. Zhang, "Design and optimization of a hybrid-driven waist rehabilitation robot," Sensors 16(12), 2121 (2016). 